\title{
An adaptive nonsingular fast terminal sliding mode control for yaw stability control of bus based on STI tire model
}

\author{
Xiaoqiang Sun ( $\nabla$ sxq@ujs.edu.cn ) \\ Jiangsu University https://orcid.org/0000-0001-6344-5582 \\ Yujun Wang \\ Jiangsu University \\ Yingfeng Cai \\ Jiangsu University \\ PakKin Wong \\ University of Macau \\ Long Chen \\ Jiangsu University
}

\section{Original Article}

Keywords: Bus, Yaw stability control, Sliding mode control, STI tire model, Co-simulation

Posted Date: October 12th, 2020

DOl: https://doi.org/10.21203/rs.3.rs-36541/v2

License: (9) This work is licensed under a Creative Commons Attribution 4.0 International License. Read Full License 


\section{Title page}

\section{An adaptive nonsingular fast terminal sliding mode control for yaw stability control of bus based on STI tire model}

Xiao-Qiang Sun, born in 1989, is currently an associate professor at Automotive Engineering Research Institute, Jiangsu University, China. He received his $\mathrm{PhD}$ degree from Jiangsu Universtiy, China, in 2016. His research interests include vehicle system dynamics. Tel: +86-15952818843; E-mail: sxq@ujs.edu.cn

Yu-Jun Wang, born in 1992, is currently a master candidate at Automotive Engineering Research Institute, Jiangsu University, China.

Ying-Feng Cai, born in 1985, is currently a professor and a $\mathrm{PhD}$ candidate supervisor at Automotive Engineering Research Institute, Jiangsu University, China. Her main research interests include vehicle system dynamics, intelligent vehicles and visual perception.

Pak-Kin Wong, is currently a professor and a $\mathrm{PhD}$ candidate supervisor at Department of Electromechanical Engineering, University of Macau, Taipa, Macau. His main research interests include automotive engines, drive trains and chassis.

Long Chen, born in 1958, is currently a professor and a PhD candidate supervisor at Automotive Engineering Research Institute, Jiangsu University, China. His main research interests include vehicle system dynamics and advanced vehicle control theory.

\section{Corresponding author: Xiao-Qiang Sun E-mail: sxq@ujs.edu.cn}


ORIGINAL ARTICLE

\section{An adaptive nonsingular fast terminal sliding mode control for yaw stability control of bus based on STI tire model}

Xiao-Qiang Sun ${ }^{1,3} \cdot$ Yu-Jun Wang ${ }^{2} \cdot$ Ying-Feng Cai ${ }^{1} \bullet$ Pak-Kin Wong $^{1} \cdot$ Long Chen $^{1}$ 
'Abstract: In this paper, a novel adaptive nonsingular fast terminal sliding mode (ANFTSM) control scheme for yaw stability control (YSC) is proposed to improve the bus curve driving stability and safety on slippery roads. There are three major contributions in the design process of the bus YSC system. The first contribution is that the STI (Systems Technologies Inc.) tire model, which can effectively reflect the coupling relationship between the tire longitudinal force and lateral force, is established based on experimental data and firstly adopted in the bus YSC system design. The second contribution is a novel YSC strategy based on ANFTSM, which has the merits of fast transient response, finite time convergence and high robustness against uncertainties and external disturbances. The third contribution is that the robust least-squares allocation method is used to solve the optimal allocation problem of the tire forces, whose objective is to achieve the desired direct yaw moment through the effective distribution of the brake force of each tire. To verify the feasibility, effectiveness and practicality of the proposed bus YSC approach, the TruckSim-Simulink co-simulation results are finally provided.

Keywords: Bus • Yaw stability control $\bullet$ Sliding mode control $\bullet$ STI tire model $\bullet$ Co-simulation

\section{Introduction}

Due to the characteristics of large quality, high center of gravity and narrow wheelbase, the bus is prone to lateral instability under special driving conditions such as turning on slippery roads [1-4]. If this lateral instability phenomenon cannot be predicted accurately and be timely and effectively controlled, it will eventually lead to bus rollover accident [5]. Therefore, it is of great significance to study the active stabilization control (ASC) strategy of bus when turning on slippery roads [6-8].

In order to improve the vehicle curve driving stability and safety on slippery roads, the yaw stability control (YSC) system has been applied to achieve the vehicle ASC function [9]. Leonardo et al. designed a direct yaw moment controller based on the combination of feedforward and feedback [10]. Chen et al. determined the desired vehicle yaw moment by means of sliding mode control [11]. Avesta et al. proposed an active controller system to supervise the vehicle lateral dynamics and then designed a differential braking yaw moment controller [12]. Ding et al. proposed a YSC strategy for in-wheel electric vehicles by using the sliding mode and nonlinear disturbance observer methods [13, 14]. Although all the above studies have made outstanding contributions to the YSC system, the vehicle tire model, which shows high

Xiao-Qiang Sun sxq@ujs.edu.cn

Automotive Engineering Research Institute, Jiangsu University, Zhenjiang, 212013, China; nonlinearity in the operation process of the ASC system [1517], is not handled effectively. Even though some studies have established the vehicle dynamics model considering the tire nonlinear characteristics, the tire model adopted cannot effectively reflect the coupling relationship between the tire longitudinal force and lateral force, which will lead to the derived YSC strategy cannot achieve good performance in practical applications.

On the basis of the accurate vehicle model, the effectiveness of the YSC control algorithm can then be guaranteed. In the existing literatures, several methodologies have been applied to improve the vehicle YSC performance [18-21]. Among them, the sliding mode control (SMC) method has been widely applied due to its strong robustness against uncertainties and disturbance. However, the traditional SMC method still has drawbacks that limit its performance in real applications, thus much effort has been devoted to finding effective solutions to eliminate those drawbacks [22-24]. Based on the conclusions of the existing research, the adaptive nonsingular fast terminal sliding mode (ANFTSM) control method, which can both obtain fast finite time convergence and chattering elimination $[25,26]$, is applied in this work to effectively solve the direct yaw moment calculation problem of bus. As is known to all, the obtained direct yaw moment needs to be further achieved by distributing the braking forces of four wheels, thus the solution of the optimal allocation problem of the tire braking forces is also crucial. In [27-29], several algorithms have been proposed to achieve well allocation of the tire forces. However, we have found that the allocation algorithms used in the current research still take a long time to calculate, thus the real-time control, which is very important for the YSC system, cannot be guaranteed reliably.

In this paper, a novel YSC scheme which both includes a new tire model applied in the vehicle system modeling, a new control algorithm used to calculate the direct yaw moment and a new method used to guarantee the solving efficiency of the tire forces allocation is presented. Therefore, the main contributions of this research are:

- The STI tire model, which can effectively reflect the coupling relationship between the tire longitudinal force and the tire lateral force, is established based on experimental data and firstly adopted in the bus YSC system design.

- The ANFTSM control algorithm, which has the merits of high robustness against uncertainty and external disturbance, fast transient response and finite time convergence, is firstly applied to solve the direct yaw moment calculation problem of the bus effectively.

- The robust least-squares control allocation method, which can effectively guarantee the solving efficiency of

State Key Laboratory of Automotive Safety and Energy, Tsinghua University, Beijing 100084, China

Department of Electromechanical Engineering, University of Macau, Taipa, Macau 
complex optimization problem like the tire longitudinal forces allocation of each tire and restrain the uncertainty in control distribution effectiveness matrix, is firstly used to solve the optimal allocation problem of the tire forces.

The rest of this paper is organized as follows: Sect. 2 presents the establishment of the STI tire model based on experimental data firstly, and on this basis, a 7-DOF nonlinear dynamics model of bus is further introduced. In Sect. 3, the YSC strategy design based on ANFTSM method is provided. Sect. 4 shows how to solve the optimal allocation problem of the tire forces by using the robust least-squares allocation method. In Sect. 5, the TruckSim-Simulink cosimulation results are presented to verify the feasibility, effectiveness and practicality of the proposed YSC approach. Finally, the conclusions and future works are given in Sect. 6.

\section{System modeling}

\subsection{STI tire model}

STI tire model is proposed by an American company, which named as "Systems Technologies Inc." [30]. Compared with other tire models, the STI tire model can not only describe the tire nonlinear mechanical characteristics accurately, but also reflect the coupling relationship between the tire longitudinal force and the tire lateral force exactly [31], thus it is very suitable for the bus YSC system design in this study. To achieve the description of the STI tire model, an important variable must be defined firstly, i.e. the composite slip coefficient $\sigma$, which is expressed as [32]:

$$
\sigma=\frac{\pi a_{p}^{2} \sqrt{k_{\alpha}^{2} \tan ^{2} \alpha+k_{s}^{2}(s / 1-s)^{2}}}{8 \mu F_{z}},
$$

where $a_{p}$ denotes the length of the tire contact patch, $k_{\alpha}$ denotes the tire lateral stiffness, $k_{s}$ denotes the tire longitudinal stiffness, $\alpha$ denotes the tire sideslip angle, $s$ denotes the tire longitudinal slip coefficient, $\mu$ is the road adhesion coefficient and $F_{z}$ is the tire vertical load. In eq. (1), the length of the tire contact patch can be further defined as [33]:

$$
a_{p}=0.0768 \sqrt{F_{z} \cdot F_{z t}} / T_{w}\left(T_{p}+5\right),
$$

where $F_{z t}$ is the tire design load, $T_{w}$ is the tire width, $T_{p}$ is the tire pressure. The tire lateral stiffness $k_{\alpha}$ and the tire longitudinal stiffness $k_{s}$ in eq. (1) can be measured by experimental tests. On this basis, to further consider the saturation effect of the tire longitudinal stiffness, a modified tire longitudinal stiffness $k_{s m}$ is defined as:

$$
k_{s m}=k_{s}+\left(k_{\alpha}-k_{s}\right) \sqrt{\sin ^{2} \alpha+\cos ^{2} \alpha},
$$

Based on the defined composite slip coefficient $\sigma$, a tire force saturation function can then be defined as:

$$
f(\sigma)=\frac{C_{1} \sigma^{3}+C_{2} \sigma^{2}+(4 / \pi) \sigma}{C_{1} \sigma^{3}+C_{3} \sigma^{2}+C_{4} \sigma+1},
$$

where $C_{1}, C_{2}, C_{3}$ and $C_{4}$ are the fixed coefficients, which can be obtained by fitting the experimental data. The function $f$ $(\sigma)$ is consistent with the mechanical properties of the tire force friction circle, thus it can reflect the tire force saturation characteristics effectively. Then, on the basis of the above equations, the standardized expressions of the tire longitudinal force and the tire lateral force of the STI tire model can be respectively described as:

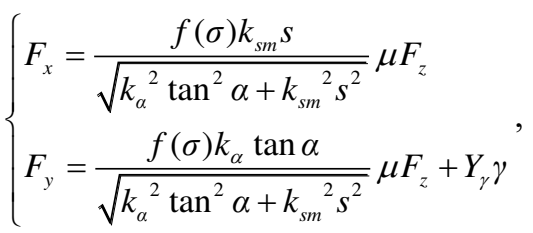

where $F_{x}$ represents the tire longitudinal force, $F_{y}$ represents the tire lateral force, $\gamma$ represents the tire camber angle, $Y_{\gamma}$ represents the tire camber coefficient, which is used to reflect the influence of the camber angle on the tire lateral force. According to eq. (5), the coupling relationship between the tire longitudinal force and the tire lateral force under compound driving conditions can be reflected exactly.

To obtain the experimental data which can accurately reflect the nonlinear mechanical characteristics of the tire, the experimental tests are conducted through a flat-plate bench assisted by the KH Automotive Technologies (Guangzhou) Co., Ltd. Figure 1 shows the experimental setup of the tire mechanical characteristics tests.
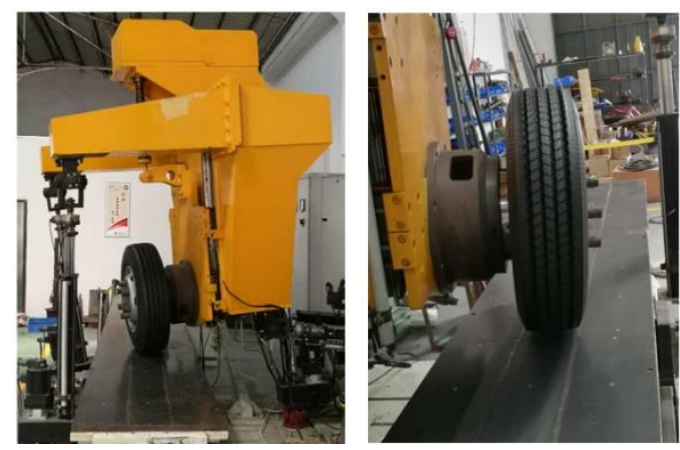

Figure 1 Experimental setup of the tire mechanical characteristics tests.

Based on the experimental data, the STI tire model parameters can then be fitted. According to the expressions of the tire forces, it is obvious that the fitting of the tire model parameters is actually to determine the fixed coefficients $C_{1}$, $C_{2}, C_{3}$ and $C_{4}$ in eq. (4). Before achieving the fitting of the fixed coefficients, the tire lateral stiffness and the tire longitudinal stiffness need to be firstly measured by experimental tests. According to the test results, the tire lateral stiffness is finally determined as $-66463 \mathrm{~N} / \mathrm{rad}$ and the tire longitudinal stiffness is finally determined as 84000 
$\mathrm{N} / \mathrm{rad}$. On this basis, the value of the composite slip coefficient $\sigma$ can then be calculated for different tire vertical loads, tire sideslip angles and tire longitudinal slip coefficients. Meanwhile, according to eq. (5) and the experimental results of the tire forces, the value of the tire force saturation function can also be determined. Therefore, by combining the value of the composite slip coefficient and the value of the tire force saturation function, the fixed coefficients $C_{1}, C_{2}, C_{3}$ and $C_{4}$ can then be determined by using the curve fitting function in the Origin software. Take the case of the tire bench test under low road adhesion coefficient, the values of the four fixed coefficients $C_{1}, C_{2}$, $C_{3}$ and $C_{4}$ are finally determined as 10, 8.98, 10 and 0 respectively.

To verify the fitting accuracy, the comparison between the experimental results and the simulation results of the tire force saturation function is shown in Figure 2. It can be seen that the experimental results of the tire force saturation function are all distributed around the fitting curve, which shows that the fitting results are highly consistent with the experimental results. Figure 3 further shows the fitting residual of the tire force saturation function. It can be seen that most of the absolute values of the fitting residual are less than 0.15 , which indicates that the fitting of the tire force saturation function in this work is trustworthy. Note that there are two other parameters that can also show the fitting effect, i.e. the R-squared and the adjusted R-squared. However, because of the residual diagram can show the fitting effect more intuitively, thus we only provide the fitting residual results in this paper.

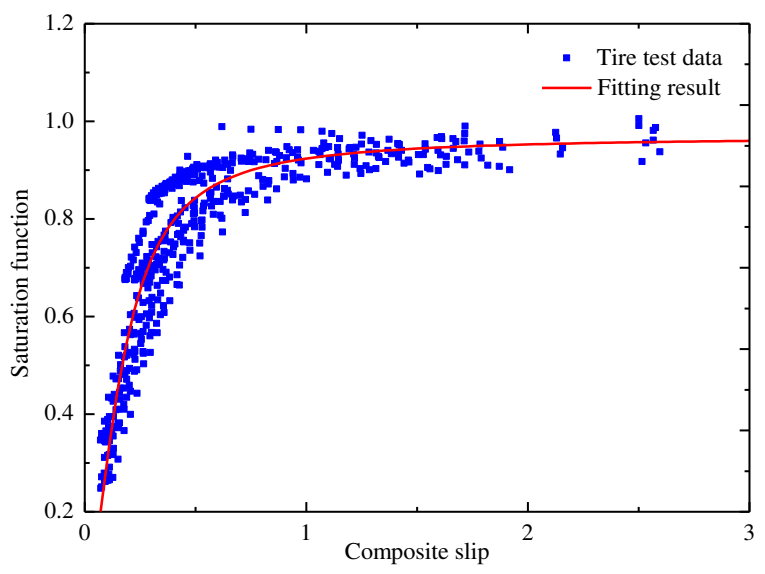

Figure 2 Comparison between the experimental results and the simulation results of the tire force saturation function.

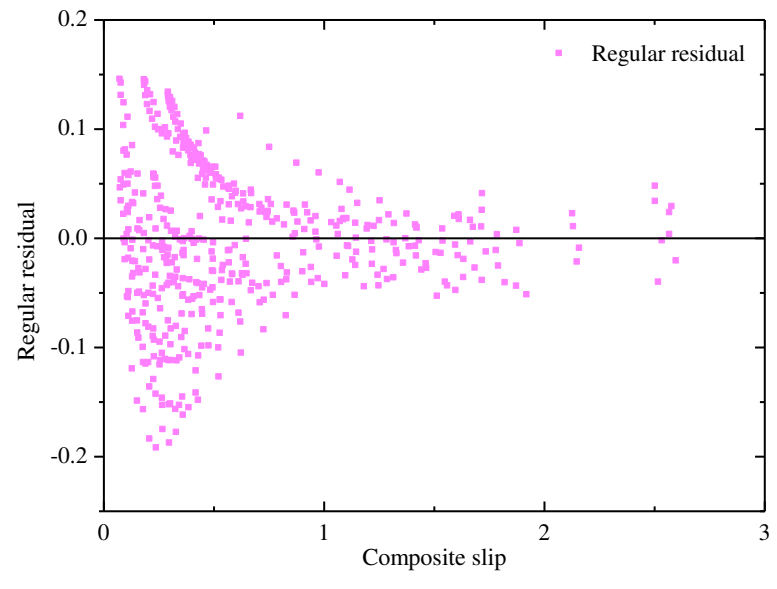

Figure 3 Fitting residual of the tire force saturation function.

\subsection{Bus dynamics model}

In this section, a 7-DOF bus dynamics model is established based on previous literatures [34-36], which includes the longitudinal motion, lateral motion, yaw motion and the rotational dynamics of four wheels. According to Newton's theorem, the dynamic equations of the bus longitudinal, lateral and yaw motions can be established as follows [37]:

$$
\left\{\begin{array}{l}
m(1 \&-r \cdot l \&)=\left(F_{x f l}+F_{x f r}\right) \cos \delta \\
-\left(F_{y f l}+F_{y f r}\right) \sin \delta+F_{x r l}+F_{x r r} \\
m\left(1 \&+r \cdot v_{x}\right)=\left(F_{x f l}+F_{x f r}\right) \sin \delta \\
+\left(F_{y f l}+F_{y f r}\right) \cos \delta+F_{y r l}+F_{y r r} \\
I_{z} \cdot \&=\left[\left(F_{x f l}+F_{x f r}\right) \sin \delta+\left(F_{y f l}+F_{y f r}\right) \cos \delta\right] a \\
+\left[\left(F_{x f r}-F_{x f l}\right) \cos \delta+\left(F_{y f l}-F_{y f r}\right) \cos \delta\right] \frac{t_{w 1}}{2} \\
+\left(F_{x r r}-F_{x r l}\right) \frac{t_{w 2}}{2}-\left(F_{y r l}+F_{y r r}\right) b
\end{array}\right.
$$

where $F_{x i j}$ and $F_{y i j}$ are the tire longitudinal and lateral forces respectively, the first subscript $i=f$ or $r$ represent front and rear axles respectively, and the second $j=l$ or $r$ denote left and right wheels. $m$ is the vehicle weight, $v_{x}$ and $v_{y}$ are the longitudinal and lateral velocities of the vehicle, $\delta$ is the front wheel steering angle, $a$ is the distance from the center of the vehicle to the front axle, $b$ is the distance from the center to the rear axle, $t_{\mathrm{w} 1}$ and $t_{\mathrm{w} 2}$ are the wheel bases of the front and rear axles, $r$ is the vehicle yaw rate. Note that the tire longitudinal and lateral forces in eq. (6) are calculated by the STI tire model which has been established in the previous section. To represent the lumped disturbance which includes the system uncertainties and external disturbance, a variable $D(t)$ which is usually assumed to be a bounded function is defined as follows:

$$
D(t) \leq \delta_{0},
$$

where $\delta_{0}$ is the upper bound of $D(t)$. 
In addition, the dynamic equations which represent the rotation of the four wheels are given by:

$$
J \cdot c_{k}=T-F_{x i j} \cdot R,
$$

where $J$ is the moment of inertia of the wheel, $\omega_{k}(k=1,2,3$, 4) represent the angular velocity of the four wheels, $R$ is the wheel radius, $T_{k}(k=1,2,3,4)$ represent the braking torques acted on the four wheels.

To more accurately reflect the vehicle load transfer effect during vehicle driving process, the tire vertical load can be calculated by combining the static tire vertical load and the load transfer effect caused by longitudinal and lateral acceleration. Thus, the related equations are expressed as [38]:

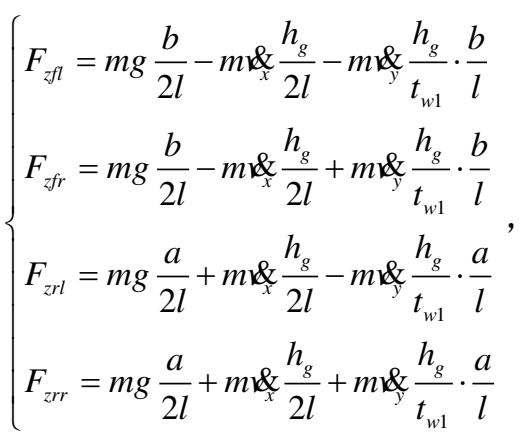

where $h_{g}$ is the height of the center of the bus, $l$ is the length of the bus. In addition, the calculation of the tire sideslip angle of each wheel is given as follows:

$$
\left\{\begin{array}{l}
\alpha_{f l}=\delta-\arctan \left(\frac{v_{y}+a r}{v_{x}-t_{w 1} r / 2}\right) \\
\alpha_{r l}=\delta-\arctan \left(\frac{v_{y}+a r}{v_{x}+t_{w 1} r / 2}\right) \\
\alpha_{r l}=-\arctan \left(\frac{v_{y}-b r}{v_{x}-t_{w 2} r / 2}\right) \\
\alpha_{r l}=-\arctan \left(\frac{v_{y}-b r}{v_{x}+t_{w 2} r / 2}\right)
\end{array},\right.
$$

Based on the above calculations for bus driving states, the wheel center speed of each wheel can then be obtained as follows $[39,40]$ :

$$
\left\{\begin{array}{l}
v_{t_{-} f l}=\left(v_{x}-\frac{t_{w 1}}{2} r\right) \cos \delta+\left(v_{y}+a r\right) \sin \delta \\
v_{t_{-} f r}=\left(v_{x}+\frac{t_{w 1}}{2} r\right) \cos \delta+\left(v_{y}+a r\right) \sin \delta \\
v_{t_{-} r l}=v_{x}-\frac{t_{w 2}}{2} r ; \quad v_{t_{-} r r}=v_{x}+\frac{t_{w 2}}{2} r
\end{array}\right.
$$

Since the angular velocity and the wheel center speed of the four wheels have been calculated, the tire longitudinal slip coefficient can then be given by:

$$
\left\{\begin{array}{l}
s_{f l}=\frac{\omega_{f l} R-v_{t_{-} f l}}{v_{t_{-} f l}}, s_{f r}=\frac{\omega_{f r} R-v_{t_{-} f r}}{v_{t_{-} f r}} \\
s_{r l}=\frac{\omega_{r l} R-v_{t_{-} r l}}{v_{t_{-} r l}}, S_{r r}=\frac{\omega_{r r} R-v_{t_{-} r r}}{v_{t_{-} r r}}
\end{array}\right.
$$

According to eqs. (9) (12), it can be seen that the tire vertical load, the tire sideslip angle and the tire longitudinal slip coefficient can be calculated by the vehicle model in real time, thus on this basis, the tire longitudinal and lateral forces can be obtained through the established STI tire model, which lays an important foundation for the following YSC system design.

\section{YSC system design based on ANFTSM}

\subsection{System overall control structure}

The main control objective of the YSC system is to ensure that the bus driving states can follow the target values calculated by the reference model when tracking curve paths on slippery roads. In this driving condition, the main challenge for calculating an optimal direct yaw moment is that the tire-road friction force cannot both meet the requirements of tire longitudinal and lateral forces. Therefore, the first objective of the YSC system is to calculate the optimal direct yaw moment by considering the coupling relationship between the tire longitudinal force and the tire lateral force. On this basis, an effective allocation algorithm should be adopted to distribute the braking forces of the four wheels, thus the optimal direct yaw moment can be achieved. In this paper, to verify the feasibility, effectiveness and practicality of the proposed YSC approach, the TruckSim vehicle model, which can accurately reflect the bus nonlinear dynamic characteristics, is used to conduct the co-simulation with Simulink. Based on the above descriptions, the system overall control structure can be shown in Figure 4.

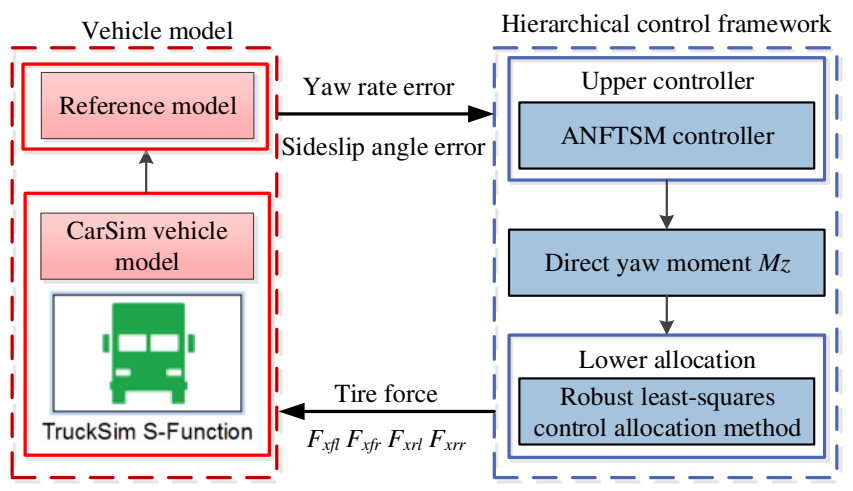

Figure 4 Hierarchical control framework. 


\subsection{YSC system design}

In this section, the ANFTSM control method is proposed to eliminate the uncertainty and disturbance in the bus YSC system design process. For general nonsingular fast terminal sliding mode (NFTSM) controller, the upper bound of disturbance is usually assumed to be known, nevertheless this assumption is difficult to be satisfied in practical application. Therefore, the ANFTSM is adopted in this paper to estimate and compensate the effects of lumped uncertainty, so as to improve the performance of the YSC control system. The block diagram of the ANFTSM control method presenting the control principle is shown in Figure 5.

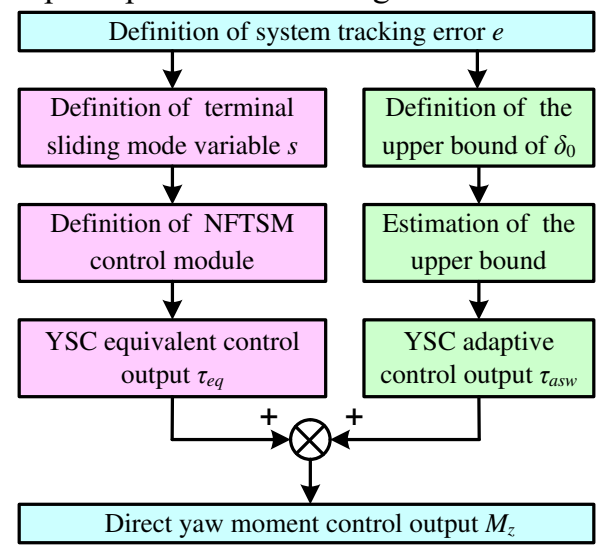

Figure 5 Control principle of ANFTSM

In the first step, according to the definition of general NFTSM, the following first-order terminal sliding mode variable is defined as [41-43]:

$$
s=e+k_{1}|e|^{\alpha_{1}} \operatorname{sign}(e)+k_{2} \mid e^{\beta_{1}} \operatorname{sign}(\&
$$

where $k_{1}$ and $k_{2}$ are positive constants, $1<\beta_{1}<2$ and $\alpha_{1}>\beta_{1}$ and $\operatorname{sign}(\cdot)$ is the signum function, $e$ represents the system tracking error, which is always expressed as:

$$
\left\{\begin{array}{l}
e=c_{1}\left(\beta-\beta_{d}\right)+\left(1-c_{1}\right)\left(\varphi-\varphi_{d}\right) \\
\&=c_{1}\left(\beta_{d}^{\&}\right)+\left(1-c_{1}\right)\left(r-r_{d}\right) \\
\& c_{1}\left(\beta_{d}\right)+\left(1-c_{1}\right)(\&-\&)
\end{array}\right.
$$

where $c_{1}$ is the weight coefficient used to determine the control specific proportion of the vehicle slip angle and the yaw rate, $\varphi$ is the vehicle yaw angle. On this basis, the time derivative of the sliding mode variable defined by eq. (13) can be obtained as:

$$
\begin{aligned}
\& & \&+\alpha_{1} k_{1}|e|^{\alpha-1} \cdot \& \beta_{1} k_{2} \mid \mathbb{Q}^{\beta-1} \cdot \& \\
& =\&+\alpha_{1} k_{1}|e|^{\alpha-1} \cdot \& \\
& +\beta_{1} k_{2} \mid \mathbb{\&}^{\beta-1} \cdot\left[c_{1}\left(\beta_{d}^{\&}\right)+\left(1-c_{1}\right)(\&-\&)\right]
\end{aligned}
$$

By further transform the expression of the vehicle yaw motion in eq. (6) as:

$$
\begin{aligned}
& \&=\frac{1}{I_{z}}\left[\frac{t_{w 1}}{2}\left(F_{y f l}-F_{y f r}\right) \sin \delta\right. \\
& \left.+a\left(F_{y f l}+F_{y f r}\right) \cos \delta-b\left(F_{y r l}+F_{y r r}\right)+M_{z}+D(t)\right]
\end{aligned}
$$

where $M_{z}$ is the direct yaw moment, which is expressed as:

$$
\begin{aligned}
& M_{z}=a\left(F_{x f l}+F_{x f r}\right) \sin \delta \\
& -\frac{t_{w 1}}{2}\left(F_{x f l}-F_{x f r}\right) \cos \delta-\frac{t_{w 2}}{2}\left(F_{x r l}-F_{x r r}\right)
\end{aligned}
$$

The lumped uncertainty in the YSC system has been proposed in eq. (16), and its upper bound can be defined as the polynomial of tracking error and its derivative, which is expressed as follows [44]:

$$
\delta_{0}=a_{0}+a_{1}|e|+a_{2} \mid \&
$$

where $a_{0}, a_{1}$ and $a_{2}$ are positive constants.

Combing eqs. (15) (17) without consideration of the uncertainty, the system control law can be expressed as:

$$
\begin{aligned}
& \&+\alpha_{1} k_{1}|e|^{\alpha-1} \cdot \& \\
& +\beta_{1} k_{2} \mid \mathbb{e}^{\beta-1} \cdot\left\{c_{1}\left(\xi_{d}^{k}\right)+\left(1-c_{1}\right)\left[\frac{1}{I_{z}}\left(P+M_{z}\right)-\&\right]\right\}=0
\end{aligned}
$$

where

$$
P=\frac{t_{w 1}}{2}\left(F_{y f l}-F_{y f r}\right) \sin \delta+a\left(F_{y f l}+F_{y f r}\right) \cos \delta-b\left(F_{y r l}+F_{y r r}\right)
$$

By satisfying eq. (19), the YSC equivalent control law can be derived as:

$$
\begin{aligned}
& \tau_{e q}=\frac{I_{z}}{1-c_{1}}\left[\left(1-c_{1}\right) \&-c_{1}\left(\alpha_{d}\right)\right. \\
& \left.-\frac{1}{\beta_{1} k_{2}} \mid d^{2-\beta_{1}} \cdot \operatorname{sign}(\&)\left(1+\alpha_{1} k_{1} \mid e^{\alpha_{1}-1}\right)\right]-P
\end{aligned}
$$

In order to reduce the influence of uncertainty on the control performance, an adaptive switching law is employed to estimate the parameters of the upper bound of system uncertainty, which is expressed as follows:

$$
\tau_{\text {asw }}=\frac{I_{z}}{1-c_{1}}\left[-k s-\left(\hat{a}_{0}+\hat{a}_{1}|e|+\hat{a}_{2} \mid \&+\eta\right) \cdot \operatorname{sign}(s)\right]
$$

where $\eta>0$ is a small positive number, $k>0$ is the switching gain, $\hat{a}_{0}, \hat{a}_{1}$ and $\hat{a}_{2}$ are the estimates of $a_{0}, a_{1}$ and $a_{2}$ respectively and the parameters are updated by the following adaptive laws: 


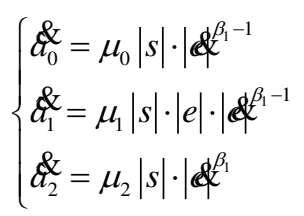

where $\mu_{0}, \mu_{1}$ and $\mu_{2}$ are arbitrary positive numbers. Therefore, by combining the equivalent control law and the adaptive control law, the overall control law of ANFTSM for the direct yaw moment is designed as follows:

$$
M_{z}=\tau_{e q}+\tau_{a s w}
$$

Based on the conclusions of the existing research, the YSC control law based on ANFTSM can effectively avoid the singular problems and ensure the fast convergence of the control system. In addition, based on Lyapunov theory, the proof process of the control system stability can be analyzed as follows [45]:

Proof. The following Lyapunov functions are defined as:

$$
V=\frac{1}{2} s^{2}+\beta_{1} k_{2} \sum_{i=0}^{2} \frac{1}{2 \mu_{i}}\left(\hat{a}_{i}-a_{i}\right)^{2}
$$

The time derivative of $V$ can then be obtained as:

$$
I^{\&}=s \& \beta_{1} k_{2} \sum_{i=0}^{2} \frac{1}{\mu_{i}}\left(\hat{a}_{i}-a_{i}\right) \&
$$

Substituting eq. (15) into eq. (26), it can be concluded that:

$$
\begin{aligned}
& I \&=s\left(\&+\alpha_{1} k_{1}|e|^{\alpha_{1}-1} \cdot \& \beta_{1} k_{2} \mid \& k^{\beta_{1}-1} \cdot\right. \\
& +\beta_{1} k_{2} \sum_{i=0}^{2} \frac{1}{\mu_{i}}\left(\hat{a}_{i}-a_{i}\right) \&
\end{aligned}
$$

By further substituting eq. (24) in eq. (27), the above formula can be rewritten as:

$$
\begin{aligned}
& I^{\&}=\beta_{1} k_{2} \mid \phi^{\beta_{1}-1}\left[D(t) s-k s^{2}-\left(\hat{a}_{0}+\hat{a}_{1}|e|+\hat{a}_{2} \mid \&+\eta\right)|s|\right] \\
& +\beta_{1} k_{2} \sum_{i=0}^{2} \frac{1}{\mu_{i}}\left(\hat{a}_{i}-a_{i}\right) c_{i}^{\&}
\end{aligned}
$$

Considering the adaptive updating laws eq. (23), eq. (28) can be rewritten as:

$$
\begin{aligned}
v^{\&} & =\beta_{1} k_{2} \mid \&^{\beta_{1}-1}\left[D(t) s-k s^{2}-\left(\hat{a}_{0}+\hat{a}_{1}|e|+\hat{a}_{2} \mid \&+\eta\right)|s|\right] \\
& +\beta_{1} k_{2}\left[\left(\hat{a}_{0}-a_{0}\right)|s| \cdot\left|\& k^{\beta_{1}-1}+\left(\hat{a}_{1}-a_{1}\right)\right| s|\cdot| e|\cdot| \&^{\beta_{1}-1}\right. \\
& \left.+\left(\hat{a}_{2}-a_{2}\right)|s| \cdot \mid \&^{\beta_{1}-1}\right]
\end{aligned}
$$

Simplifying eq. (29) yields to the following formula:

$$
\&=\beta_{1} k_{2} \mid \&^{\beta_{1}-1}\left[D(t) s-\eta|s|-k s^{2}-\left(a_{0}+a_{1}|e|+a_{2}|\&| s \mid\right]\right.
$$

Combining eq. (7) and eq. (18), the system Lyapunov function satisfies:

$$
\begin{aligned}
I & \leq \beta_{1} k_{2}|\&|^{\beta_{1}-1}\left[D(t)|s|-\eta|s|-k s^{2}-\left(a_{0}+a_{1}|e|+a_{2}|\&| s \mid\right]\right. \\
& \leq \beta_{1} k_{2}|\&|^{\beta_{1}-1}\left[-\eta|s|-k s^{2}\right] \leq 0
\end{aligned}
$$

According to the above analysis, it can be seen that the system state can asymptotically converge to zero along the sliding surface $s=0$ in finite time, which proves the stability of the proposed ANFTSM control system.

\section{Optimal allocation of tire braking force}

\subsection{Description of the dynamic allocation problem}

The main objective of the dynamic allocation problem is to determine the optimal braking forces of the four wheels, thus the obtained direct yaw moment can be achieved [46-49]. However, it is obvious that the coupling relationship between the tire longitudinal force and the tire lateral force must be satisfied for a single wheel in the dynamic allocation process, thus the motion stability of the bus when tracking curve paths on slippery roads can be guaranteed. It is obvious that this treatment will make the dynamic allocation problem more difficult when considering the tire force constraints. Therefore, how to achieve the dynamic allocation of all the tire forces to achieve the desired yaw moment is a challenging research task [50-52]. In this work, considering the uncertainty in the tire force allocation problem and the problem-solving efficiency, the robust least-squares allocation method is utilized to solve the optimal allocation problem of the tire braking forces.

Based on the theory of robust least-squares allocation algorithm, an optimization objective needs to be solved, that is, eq. (17) should be firstly transformed into $B u=v$ without considering the uncertainty and external disturbance. In this allocation rule, $v$ is the desired yaw moment $M_{z}, u$ is the actuator response, i.e. the braking forces of four wheels and $B$ is the efficiency matrix. This treatment linearizes the relationship between $u$ and $v$, and hence, the efficiency matrix $B$ can be written as:

$$
B=\left[\begin{array}{llll}
-\frac{t_{w 1}}{2} & \frac{t_{w 1}}{2} & -\frac{t_{w 2}}{2} & \frac{t_{w 2}}{2}
\end{array}\right]
$$

\subsection{Weighted least-squares allocation method}

Firstly, a reasonable objective function of the tire forces allocation should be defined, which is described as follows:

$$
\min _{u} J=u^{T} W u
$$


where $W$ is a diagonal matrix which considers the utilization ratio of the tire forces, whose element are given as follows:

$$
w_{i j}=\frac{1}{\left(\mu_{i j} F_{z i j}\right)^{2}}
$$

where $F_{z i j}$ is the tire vertical load and $\mu_{i j}$ is the road adhesion coefficient.

The essence of the optimization objective for eq. (33) is to minimize the control energy consumption of the actuator, i.e. minimizing the braking force of the tire, so as to maintain a low tire utilization ratio and improve the stability margin of the tire. In order to simplify the operation process and reduce the calculation time, the above optimization problem can be further rewritten as follows:

$$
\min J=\left\|W_{v}(B u-v)\right\|_{2}^{2}+\left\|W_{u}\left(u-u_{d}\right)\right\|_{2}^{2}
$$

where $W_{v}$ and $W_{u}$ are the weight matrix for the desired yaw moment and the tire braking forces respectively, and $u_{d}$ is the target control quantity of the actuator.

\subsection{Robust least-squares allocation method}

Although the weighted least-squares method is fast in calculation without considering the system uncertainty. However, in the case of uncertainty and external disturbance, this simplified method will reduce the allocation efficiency. Therefore, when the lumped uncertainty is fully considered, an uncertainty efficiency matrix $\Delta B$ should be introduced. In conclusion, the allocation error problem in eq. (35) can then be transformed into the following robust least-squares allocation problem:

$$
u_{R L S C A}=\arg \min \max _{\|\Delta B\|_{\infty} \leq \rho}\|(B+\Delta B) u-v\|
$$

where $\Delta B$ is the efficiency matrix used to reflect the system lumped uncertainty.

In order to solve this problem, the robust least-squares control allocation method is used, and then the allocation problem can be transformed into a second-order cone programming problem. In the first, when the variable $u$ is in the definition field, a worst case residual can be defined as:

$$
r(u)=\max _{\|\Delta B\|_{\infty} \leq \rho}\|(B+\Delta B) u-v\|
$$

According to the triangle inequality transformation, the above equation can be rewritten as:

$$
r(u) \leq \max _{\|\Delta B\|_{\infty} \leq \rho}(\|B u-v\|+\|\Delta B u\|)=\|B u-v\|+\max _{\|\Delta B\|_{\infty} \leq \rho}\|\Delta B u\|
$$

Then, the following assumptions can be given as:

$$
\Delta B=\frac{\rho}{\|u\|} \varepsilon u^{\mathrm{T}}
$$

where

$$
\varepsilon=\left\{\begin{array}{l}
\frac{B u-v}{\|B u-v\|}, \quad B u \neq v \\
\text { unit norm vector, otherwise }
\end{array}\right.
$$

Based on the above assumptions, the worst-case residual can be redefined as:

$$
r(u)=\|B u-v\|+\rho\|u\| \leq \lambda
$$

where $\lambda$ is the upper bound of the minimum residual for the optimal solution of variable $u$ in the definition domain.

In conclusion, the robust least-squares problem is then transformed into the following second-order cone optimization problem (SOCP):

$$
\begin{gathered}
\min _{u, \tau, \lambda} \lambda \\
\text { s.t. }\left\{\begin{array}{l}
\|B u-v\| \leq \lambda-\tau \\
\rho\|u\| \leq \tau \\
\underline{u} \leq u \leq \bar{u}
\end{array}\right.
\end{gathered}
$$

where $\tau$ is a real number, and $\lambda>\tau$ is satisfied. On this basis, the optimal solution $u_{R L S C A}$ to the aforementioned allocation problem shown in eq. (42) can be given by [53]:

$$
u_{R L S C A}= \begin{cases}\left(\mu I+B^{T} B\right)^{-1} B^{\mathrm{T}} v, & \text { if } \mu=\frac{(\lambda-\tau) \tau \rho^{2}}{\tau^{2}+\rho^{2} s}>0 \\ B^{+}, & \text {else }\end{cases}
$$

\section{Simulation results and analyses}

In this section, the effectiveness of the proposed YSC approach for bus is verified by simulation results. Since the YSC system is only activated under those driving conditions in which the tire-road friction force cannot both meet the requirements of the tire longitudinal and lateral forces, thus in this work, two simulation cases in which the road adhesion coefficients are 0.1 and 0.3 respectively are provided. By conducting efficient simulation comparisons, the parameters of the ANFTSM control system are as follows: $c_{1}=0.5, \alpha_{1}=2$, $\beta_{1}=5 / 3, k_{1}=1, k_{2}=1, k=50, \eta=0.5, \mu_{0}=0.01, \mu_{1}=0.01, \mu_{2}=0.01$. In the co-simulation process, the vehicle is assumed to track the double lane curve path on two slippery roads at fixed speed $(35 \mathrm{~km} / \mathrm{h})$ and the target trajectory curve is shown in Figure 6. The vehicle parameters adopted in the TruckSimSimulink co-simulation are given in Table 1.

Table 1 Bus simulation parameters

\begin{tabular}{cc}
\hline Parameters & Value \\
\hline Total mass of bus, $m(\mathrm{~kg})$ & 10900 \\
Moment of inertia, $I_{\mathrm{z}}\left(\mathrm{kg} \cdot \mathrm{m}^{2}\right)$ & 31200 \\
Longitudinal distance of cog to front axle, $a(\mathrm{~m})$ & 5.4 \\
Longitudinal distance of cog to front axle, $b(\mathrm{~m})$ & 5.1 \\
Front wheel tread, $t_{\mathrm{w} 1}(\mathrm{~m})$ & 2.2 \\
Rear wheel tread, $t_{\mathrm{w} 2}(\mathrm{~m})$ & 2.2
\end{tabular}




$\begin{array}{cc}\text { Front tire cornering stiffness, } k_{\mathrm{f}}(\mathrm{N} / \mathrm{rad}) & -6000 \\ \text { Moment of inertia of the wheel, } J\left(\mathrm{~kg} \cdot \mathrm{m}^{2}\right) & 65 \\ \text { Wheel radius, } R(\mathrm{~m}) & 0.52 \\ \text { Height of vehicle center, } h_{\mathrm{g}}(\mathrm{m}) & 1.35\end{array}$

\subsection{Simulation results of the first case $(\mu=0.1)$}

The simulation results comparison of the vehicle slip angle, the vehicle yaw rate and the obtained direct yaw moment between the ANFSTM and the SMC are shown in Figure 7. As can be seen from Figure 7(a) and Figure 7(b), the peak values of the vehicle slip angle and the vehicle yaw rate controlled by the ANFTSM are decreased significantly compared with that controlled by the conventional SMC, among of which the absolute value of the vehicle slip angle peak value is reduced from $0.05 \mathrm{rad}$ to $0.03 \mathrm{rad}$ and the absolute value of the vehicle yaw rate peak value is reduced from $0.35 \mathrm{rad}$ to $0.25 \mathrm{rad}$, which demonstrates the feasibility, effectiveness and practicality of the proposed YSC approach for vehicle tracking curve path on slippery road. Figure 6(c) shows the difference of the direct yaw moment between the ANFSTM and the SMC. It is obviously that the direct yaw moment calculated by the two methods is different, which illustrates that the proposed YSC approach in this work can effectively calculate the optimal direct yaw moment by considering the tire force constraints on slippery road.

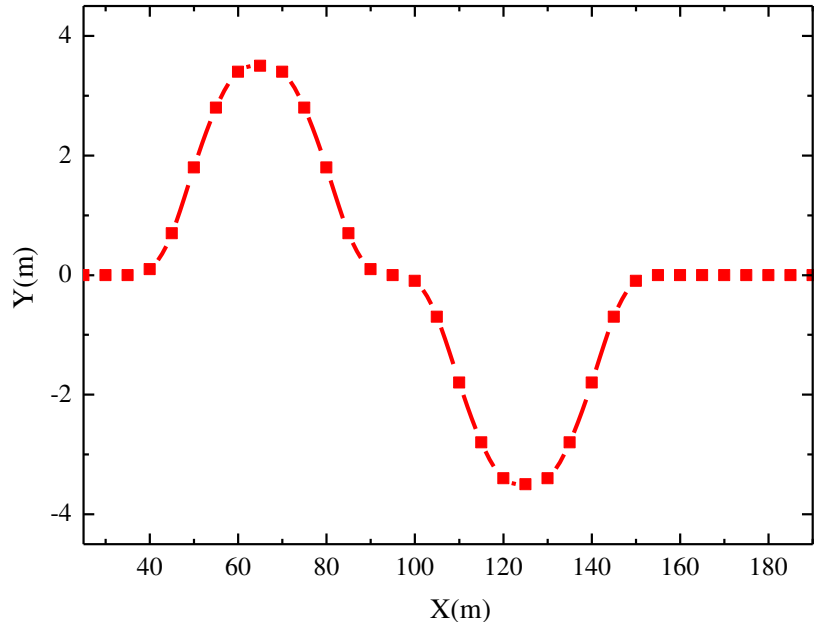

Figure 6 Target trajectory curves.

To achieve the direct yaw moment and guarantee the path tracking accuracy, the optimal allocation of the tire braking forces are also conducted. Figure 8 shows the allocation

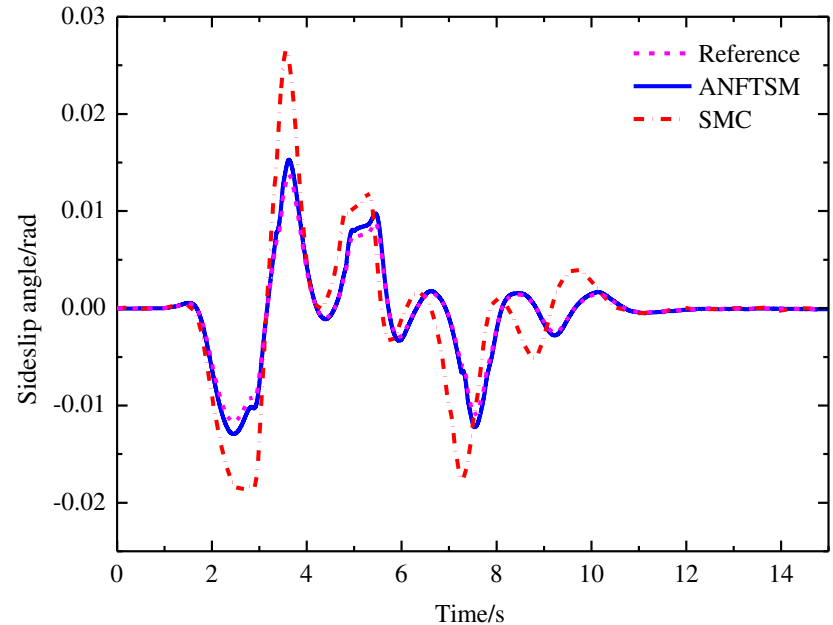

(a) Comparison of the vehicle slip angle

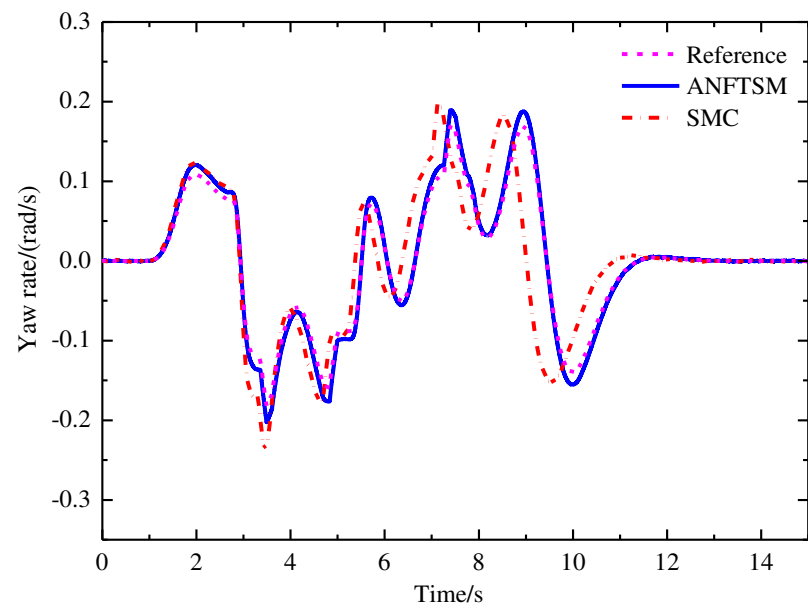

(b) Comparison of the vehicle yaw rate

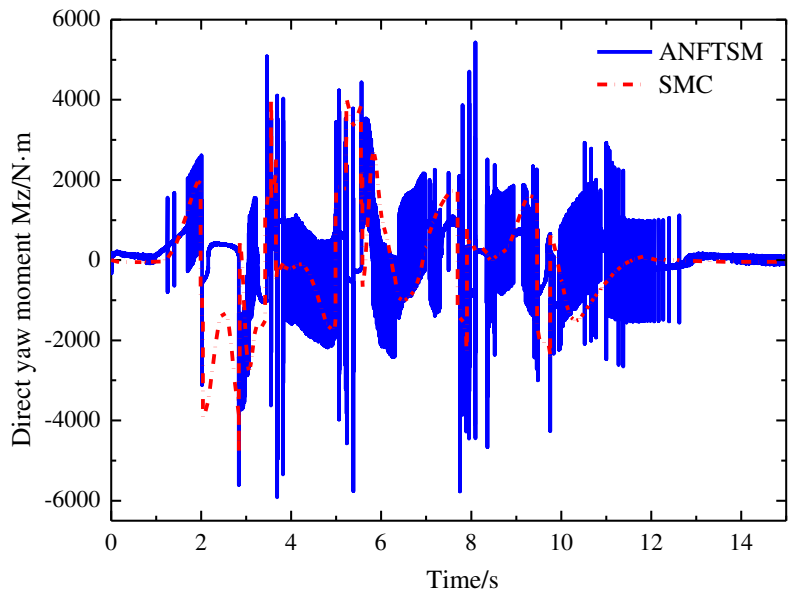

(c) Comparison of the calculated direct yaw moment

Figure 7 Simulation results comparison between the ANFTSM and the $\operatorname{SMC}(\mu=0.1)$.

results of the tire braking forces of the four wheels. It can be seen that different tire forces are optimally allocated to achieve the direct yaw moment. In addition, as shown in the figures, when the vehicle is steering, the absolute values of 
the tire longitudinal forces of the left-front wheel and the leftrear wheel are large, while that of the right-front wheel and the right-rear wheel are small. This tire forces allocation results can not only realize the additional yaw moment, but also ensure that the overall load rate of the four wheels is maintained at a low level, so that the vehicle will not lose lateral stability.
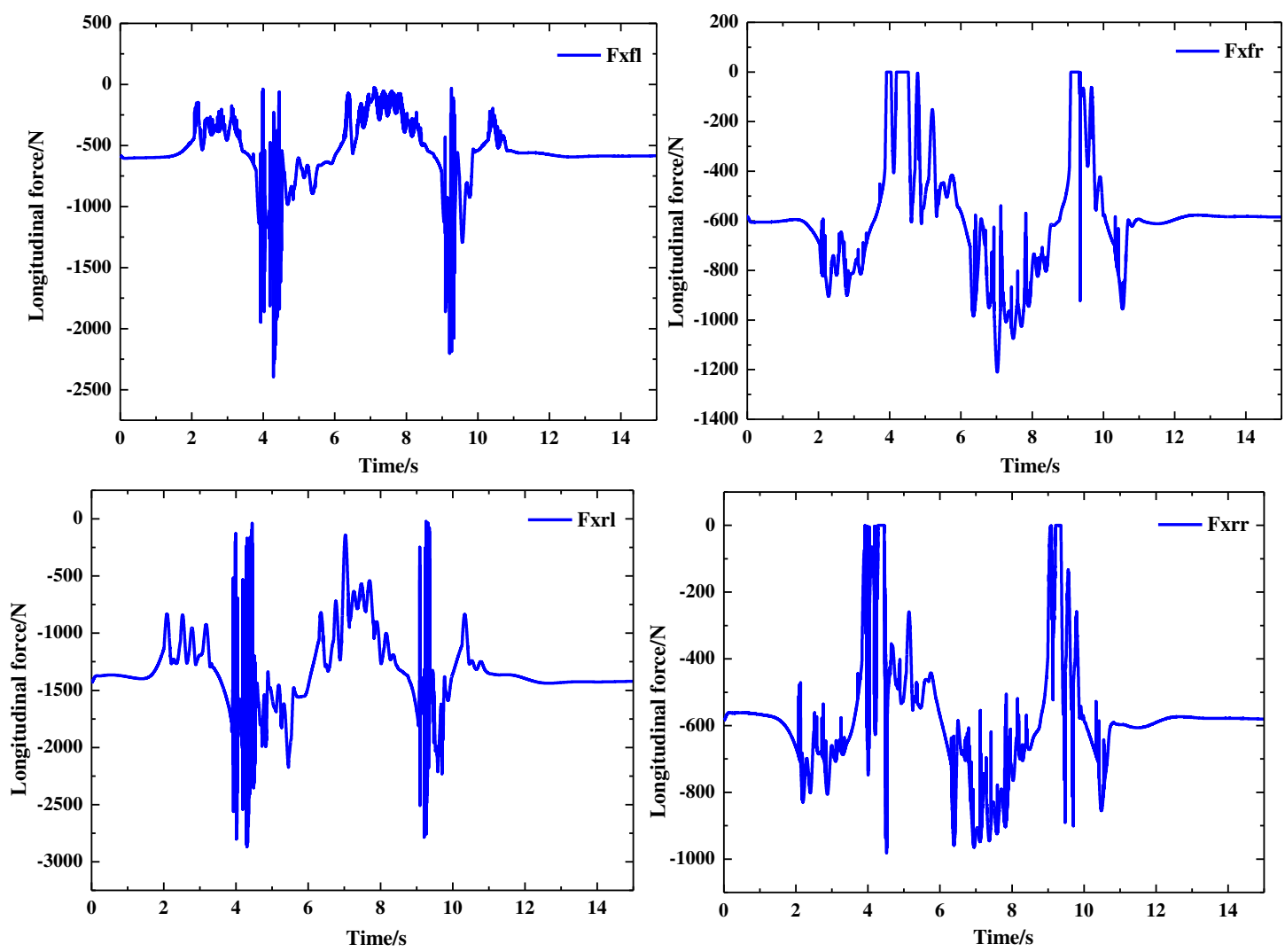

Figure 8 Tire forces allocation results based on the robust least-squares control allocation method $(\mu=0.1)$

\subsection{Simulation results of the second case $(\mu=0.3)$}

Similarly, the simulation results comparison of the vehicle slip angle, the vehicle yaw rate and the obtained direct yaw moment between the ANFSTM and the SMC for the second case are shown in Figure 9. As can be seen from Figure 9(a) and Figure 9(b), the peak values of the vehicle slip angle and the vehicle yaw rate controlled by the NFTSM are also decreased significantly compared with that controlled by the conventional SMC, among of which the absolute value of the vehicle slip angle peak value is reduced from $0.025 \mathrm{rad}$ to $0.015 \mathrm{rad}$ and the absolute value of the vehicle yaw rate peak value is reduced from $0.25 \mathrm{rad}$ to $0.2 \mathrm{rad}$.

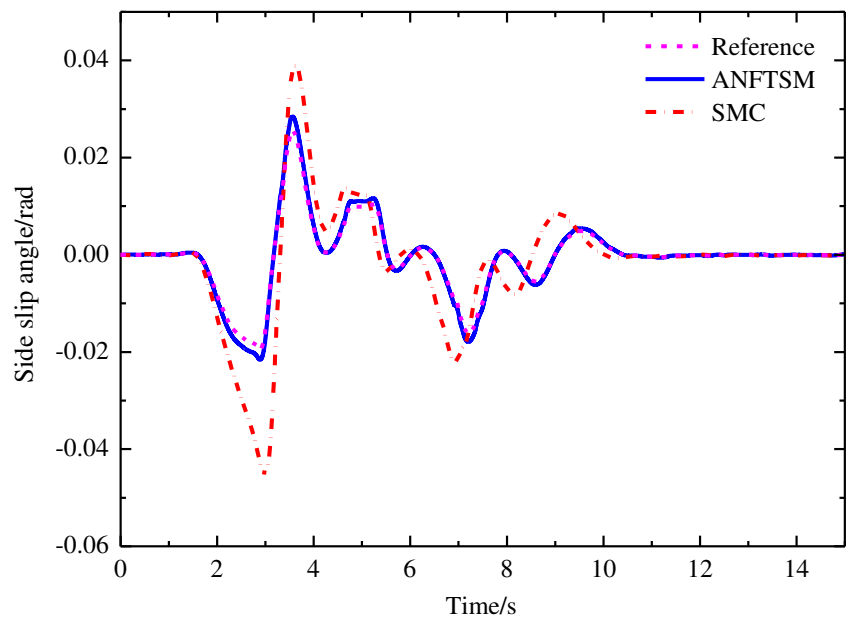

(a) Comparison of the vehicle slip angle 


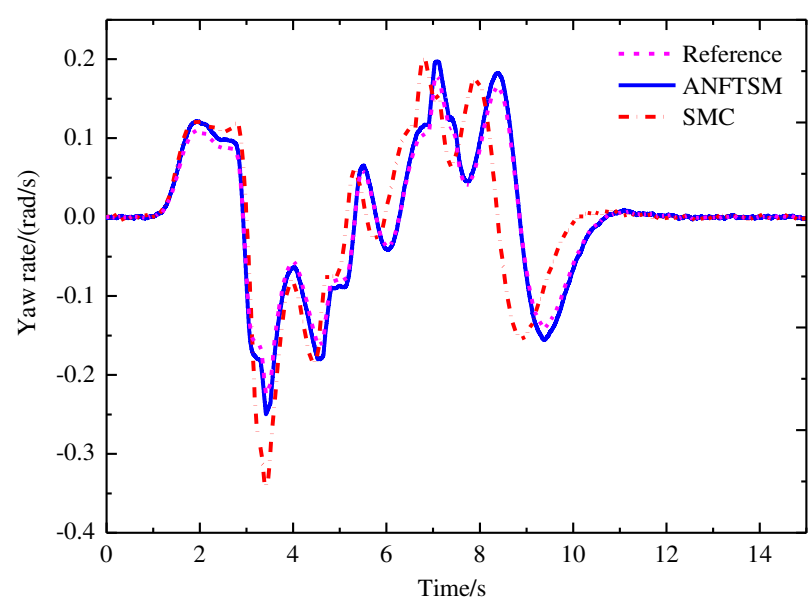

(b) Comparison of the vehicle yaw rate
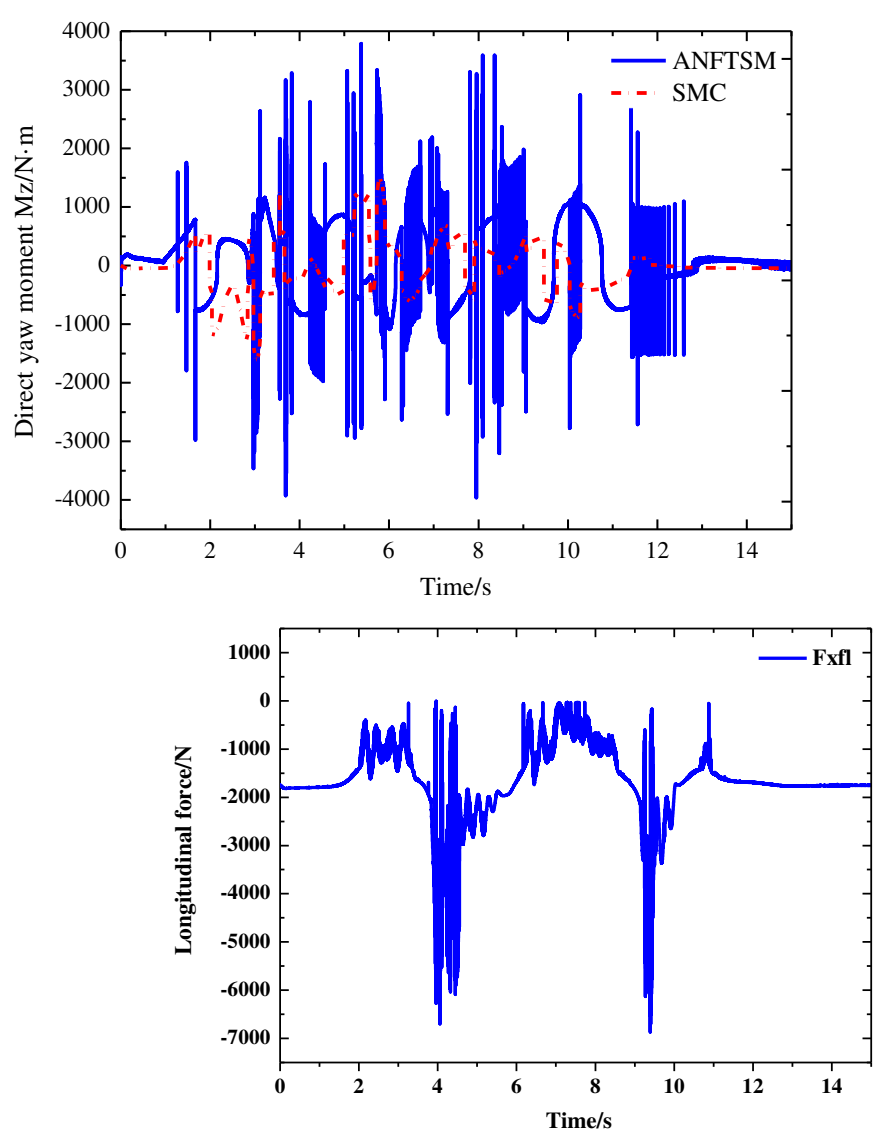

(c) Comparison of the calculated direct yaw moment

Figure 9 Simulation results comparison between the ANFTSM and the $\operatorname{SMC}(\mu=0.3)$.

The allocation results of the tire braking forces of the four wheels in the second case are shown in Figure 10. Compared with the allocation results of the tire forces shown in Figure 10 , it can be seen that the tire forces of the four wheels in the second case are obviously larger than that in the first case, which is due to the larger road adhesion coefficients. In this case, the tire-road friction force increases. This phenomenon shows that the optimal allocation of the tire forces based on the robust least-squares control allocation method can effectively reflect the relationship between the tire longitudinal force and the tire lateral force and the tire force constraints on slippery road, thus the resulted tire forces can then be achieved through the actuators of the bus braking system in practical applications.

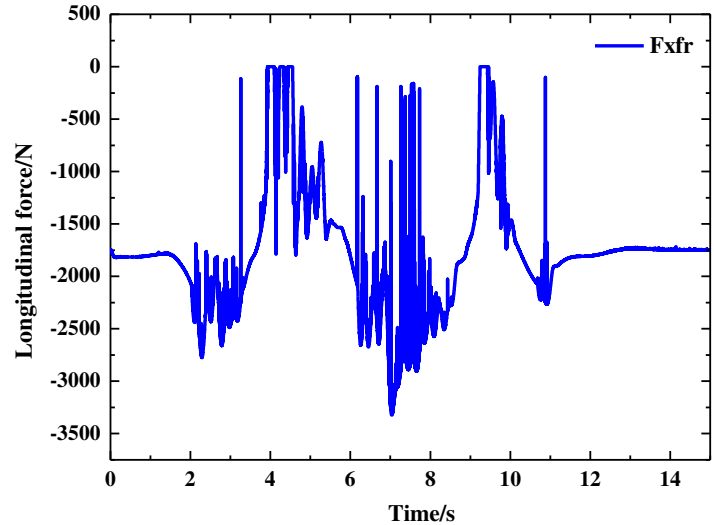



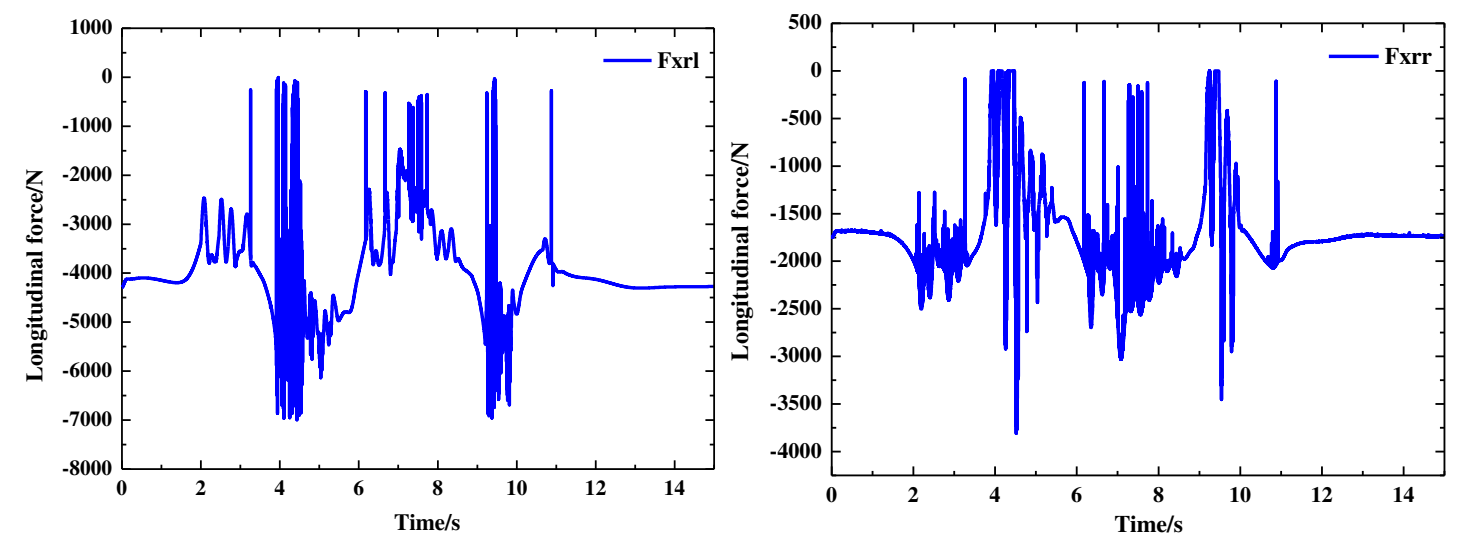

Figure 10 Tire forces allocation results based on the robust least-squares control allocation method $(\mu=0.3)$ 


\section{Conclusions}

In this paper, a novel bus YSC scheme to improve the curve driving stability and safety on slippery roads is proposed. According to the experimental data, the STI tire model, which can effectively reflect the coupling relationship between the tire longitudinal force and the tire lateral force, is established and firstly adopted in the bus YSC system design. On this basis, the ANFTSM control algorithm is applied to solve the direct yaw moment calculation problem, and the optimal allocation of the tire forces is further conducted using the robust least-squares allocation method to achieve the derived direct yaw moment. TruckSimSimulink co-simulation results are finally provided to verify the feasibility, effectiveness and practicality of the proposed YSC approach. All the co-simulation results show that the peak values of the bus slip angle and the bus yaw rate controlled by the ANFTSM are decreased significantly compared with that controlled by the conventional SMC, which demonstrates the vehicle motion stability when tracking curve paths on slippery roads is improved by using the ANFTSM method. In addition, the optimal allocation of the tire forces can be effectively achieved by the robust leastsquares allocation method, in which the coupling relationship between the tire longitudinal force and the tire lateral force and the tire force constraints on slippery road are reflected accurately. Therefore, this work is essential for moving forward the scientific and industrial knowledge on vehicle YSC system design.

\section{Declaration}

\section{Funding}

Supported by the National Natural Science Foundation of China (Grant Nos. 52072161, 51705207), the China Postdoctoral Science Foundation (Grant No. 2019T120398), the State Key Laboratory of Automotive Safety and Energy (Grant No. KF2016), the Vehicle Measurement Control and Safety Key Laboratory of Sichuan Province (Grant No. QCCK2019-002) and the Young Elite Scientists Sponsorship Program by CAST (Grant No.2018QNRC 001).

\section{Availability of data and materials}

The datasets supporting the conclusions of this article are included within the article.

\section{Authors' contributions}

The author' contributions are as follows: Xiao-Qiang Sun was in charge of the whole trial; Yu-Jun Wang wrote the manuscript; Ying-Feng Cai, Pak-Kin Wong, Xing $\mathrm{Xu}$ and
Long Chen assisted with sampling and laboratory analyses.

\section{Competing interests}

The authors declare no competing financial interests.

\section{Consent for publication}

Not applicable

\section{Ethics approval and consent to participate}

Not applicable

\section{References}

[1] S. Tian, L. Wei, C. Schwarz, et al. An earlier predictive rollover index designed for bus rollover detection and prevention $[\mathrm{J}]$. Journal of Advanced Transportation, 2018, 2713868.

[2] M. Ghazali, M. Durali, H. Salarieh. Vehicle trajectory challenge in predictive active steering rollover prevention $[\mathrm{J}]$. International Journal of Automotive Technology, 2017, 18(3): 511-521.

[3] H. Termous, H. Shraim, R. Talj, et al. Coordinated control strategies for active steering, differential braking and active suspension for vehicle stability, handling and safety improvement [J]. Vehicle System Dynamics. 2019, 57(11): 1494-1529.

[4] Xu. X, Mi. J, Wang. F, et al. Design of differential braking control system of travel trailer based on multi-objective PID [J]. Journal of Jiangsu University (Natural Science Edition). 2020, 41(2): 172180 .

[5] J. Bai, G. Meng, W. Zuo. Rollover crashworthiness analysis and optimization of bus frame for conceptual design [J]. Journal of Mechanical Science and Technology, 2019, 33(7): 3363- 3373.

[6] T. Chen, X. Xu, L. Chen, et al. Estimation of longitudinal force, lateral vehicle speed and yaw rate for four-wheel independent driven electric vehicles, Mechanical Systems and Signal Processing. 2018, 101: 377-388.

[7] T. Chen, L. Chen, X. Xu, et al. Reliable sideslip angle estimation of four-wheel independent drive electric vehicle by information iteration and fusion, Mathematical Problems in Engineering. 2018, 2018: 9075372.

[8] Ma. Y, Zhang. Z, Z. Niu, et al. Design and verification of integrated control strategy for tractor-semitrailer AFS/DYC, Journal of Jiangsu University (Natural Science Edition). 2018, 39(5): 530-536.

[9] H. Zhang, J. Liang, H. Jiang, et al. Stability research of distributed drive electric vehicle by adaptive direct yaw moment control, IEEE Access. 2019, 7: 106225-106237.

[10] L. D. Novellis, A. Sorniotti, P. Gruber, et al. Direct yaw moment control actuated through electric drivetrains and friction brakes: Theoretical design and experimental assessment. Mechatronics. 2015, 26:1-15.

[11] Y. Chen, J. Hedrick, K. Guo. A novel direct yaw moment controller for in-wheel motor electric vehicles, Vehicle System Dynamics. 2013, 51(6): 925-942.

[12] A. Goodarzi, F. Diba, E. Esmailzadeh. Innovative active vehicle safety using integrated stabilizer pendulum and direct yaw moment control, Journal of Dynamic Systems, Measurement, and Control. 2014: 136(5): 051026.

[13] S. Ding, J. Sun, Direct yaw-moment control for 4WID electric vehicle via finite-time control technique, Nonlinear Dynamics. 2017, 88(1): 239-254.

[14] S. Ding, L. Liu, W. Zheng, Sliding mode direct yaw-moment 
control design for in-wheel electric vehicles, IEEE Transactions on Industrial Electronics. 2017, 64(8): 6752-6762.

[15] W. Huang, P. Wong, K. Wong, et al. Adaptive neural control of vehicle yaw stability with active front steering using an improved random projection neural network, Vehicle System Dynamics. DOI: $10.1080 / 00423114.2019 .1690152$.

[16] Sun. X, Hu. W, Cai. Y, et al. Identification of a piecewise affine model for the tire cornering characteristics based on experimental data, Nonlinear Dynamics. 2020, 101(2): 857-874.

[17] Sun. X, Cai. Y, Wang. S, et al. Piecewise affine identification of tire longitudinal properties for autonomous is driving control based on data-driven, IEEE Access. 2018, 6: 47424-47432.

[18] Y. Shi, Q. Liu, F. Yu, Design of an adaptive FO-PID controller for an in-wheel-motor driven electric vehicle, SAE International Journal of Commercial Vehicles. 2017, 10: 265-274.

[19] H. Guo, F. Liu, F. Xu, et al. Nonlinear model predictive lateral stability control of active chassis for intelligent vehicles and its FPGA implementation, IEEE Transactions on Systems Man Cybernetics-systems. 2019, 49(1): 2-13.

[20] Q. Meng, T. Zhao, C. Qian, et al. Integrated stability control of AFS and DYC for electric vehicle based on non-smooth control, International Journal of Systems Science. 2018, 49(7): 1518-1528.

[21] J. Song, Development and comparison of integrated dynamics control systems with fuzzy logic control and sliding mode control, Journal of Mechanical Science and Technology. 2013, 27(6): 1853-1861.

[22] J. Wang, R. He. Hydraulic anti-lock braking control strategy of a vehicle based on a modified optimal sliding mode control method, P. I. Mech. Eng. D-Journal of Automobile Engineering. 2019, 233(12): 3185-3198.

[23] X. Sun, Y. Cai, C. Yuan, et al. Fuzzy sliding mode control for the vehicle height and leveling adjustment system of an electronic air suspension, Chinese Journal of Mechanical Engineering. 2018, 31(1): 25 .

[24] S. Chen, J. Wang, M. Yao, et al. Improved optimal sliding mode control for a non-linear vehicle active suspension system, Journal of Sound and Vibration. 2017, 395: 1-25.

[25] Z. Yang, D. Zhang, X. Sun, et al. Nonsingular fast terminal sliding mode control for a bearingless induction motor, IEEE Access. 2017, 5: 16656-16664.

[26] E. Mousavinejad, Q. Han, F. Yang, et al. Integrated control of ground vehicles dynamics via advanced terminal sliding mode control, Vehicle System Dynamics. 2017, 55(2): 268-294.

[27] A. Asiabar, R. Kazemi. A direct yaw moment controller for a four in-wheel motor drive electric vehicle using adaptive sliding mode control, P. I. Mech. Eng. K- Journal of Multi-body Dynamics. 2019, 233(3): 549-567.

[28] J. Zhang, J. Li. Integrated vehicle chassis control for active front steering and direct yaw moment control based on hierarchical structure, Transactions of the Institute of Measurement and Control. 2019, 41(9): 2428-2440.

[29] S. Yue, Y. Fan. Hierarchical direct yaw-moment control system design for in-wheel motor driven electric vehicle, International Journal of Automotive Technology. 2018, 19(4): 695-703.

[30] J. Wagner, J. Keane. A strategy to verify chassis controller software-dynamics, hardware, and automation, IEEE Transactions on Systems Man \& Cybernetics. 1997, 27(4): 480-493.

[31] M. Reiter, J. Wagner. Automated automotive tire inflation systemeffect of tire pressure on vehicle handling, IFAC. Proceedings. 2010, 43(7): 638-643.

[32] K. Pan, Y. Lu. Analysis on vehicle dynamic simulating STI tire model used in driving simulator, Auto Engineer. 2009, 2: 28-30.

[33] Q. Xia, L. Chen, X. Xu, et al. Running states estimation of autonomous four-wheel independent drive electric vehicle by virtual longitudinal force sensors, Mathematical Problems in Engineering. 2019, 2019: 8302943.

[34] J. Tian, J. Tong, S. Luo. Differential steering control of four-wheel independent-drive electric vehicles, Energies. 2018, 11(11): 2892.

[35] Liu. T, Tang. X, Wang. H, et al. Adaptive hierarchical energy management design for a plug-in hybrid electric vehicle. IEEE Transactions on Vehicular Technology, 2019, 68(12): 1151311522.

[36] Chen. L, Chen. T, Xu. X, et al. Sideslip angle estimation of inwheel motor drive electric vehicles by cascaded multi-Kalman filters and modified tire model, Metrology and Measurement Systems, 2019, 26(1): 185-208.

[37] T. Chen, X. Xu, Y. Li, et al. Speed-dependent coordinated control of differential and assisted steering for in-wheel motor driven electric vehicles, P. I. Mech. Eng. D- Journal of Automobile Engineering. 2018, 232(9): 1206-1220.

[38] L. Chen, T. Chen, X. Xu, et al. Multi-objective coordination control strategy of distributed drive electric vehicle by orientated tire force distribution method, IEEE Access. 2018, 6: 69559-69574.

[39] X. Zhang, H. He, J. Nie, et al. Performance analysis of semi-active suspension with skyhook-inertance control, Journal of Jiangsu University (Natural Science Edition). 2018, 39(5): 497-502.

[40] Qin. Y, Tang. X, Jia. T, et al. Noise and vibration suppression in hybrid electric vehicles: state of the art and challenges. Renewable and Sustainable Energy Reviews, 2020, 124: 109782.

[41] S. Jiang, P. Wong, R. Guan, et al. An efficient fault diagnostic method for three-phase induction motors based on incremental broad learning and non-negative matrix factorization, IEEE Access. 2019, 7: 17780-17790.

[42] Ye. H, Li. G, Ding. S, et al. Direct yaw moment control of electric vehicle based on non-smooth control technique, Journal of Jiangsu University (Natural Science Edition). 2018, 39(6): 640-646.

[43] Wang. J, Chen. S, Jing. T, et al. Remote irrigation control system for soilless culture, Journal of Drainage and Irrigation Machinery Engin, 2020, 38(9): 959-965.

[44] S. Ding, L. Liu, J. H. Park. A novel adaptive nonsingular terminal sliding mode controller design and its application to active front steering system, International Journal of Robust and Nonlinear Control. 2019, 29(12): 4250-4269.

[45] S. Ding, W. Zheng, Nonsingular terminal sliding mode control of nonlinear second-order systems with input saturation, International Journal of Robust and Nonlinear Control. 2016, 26(9): 1857-1872.

[46] H. Jiang, F. Cao, W. Zhu. Control method of intelligent vehicles cluster motion based on SMC, Journal of Jiangsu University (Natural Science Edition). 2018, 39(4): 385-390.

[47] Geng. G, Wei. B, Duan. C, et al. A strong robust observer of distributed drive electric vehicle states based on strong trackingiterative central difference Kalman filter algorithm, Advances in Mechanical Engineering, 2018, 10(6): 1687814018779682.

[48] Liu. J, Gao. L, Zhang. J, et al. Super-twisting algorithm secondorder sliding mode control for collision avoidance system based on active front steering and direct yaw moment control, P. I. Mech. Eng. D- Journal of Automobile Engineering. 2020: 0954407020948298.

[49] P. Sathishkumar, Wang. R, Yang. L, et al. Trajectory control for tire burst vehicle using the standalone and roll interconnected active suspensions with safety-comfort control strategy, Mechanical Systems and Signal Processing, 2020, 142: 106776.

[50] B. Xu, G. Shi, W. Ji, et al. Design of an adaptive nonsingular terminal sliding model control method for a bearingless permanent magnet synchronous motor, Transactions of the Institute of Measurement and Control. 2017, 39(12): 1821-1828.

[51] Li. Y, Xu. X, Wang. W. GA-BPNN based hybrid steering control 
approach for unmanned driving electric vehicle with in-wheel motors, Complexity, 2018, 6132139.

[52] J. Wang, X. Yu, Z. Hui, et al. Influence of running speed and lateral distance on vehicle transient aerodynamic characteristics during curve crossing, Journal of Jiangsu University (Natural Science Edition). 2017, 38(3): 249-253.

[53] J. Erway, P. Gill. A subspace minimization method for the trustregion step, SIAM Journal on Optimization. 2009, 20(3): 14391461.

\section{Biographical notes}

Xiao-Qiang Sun, born in 1989, is currently an associate professor at Automotive Engineering Research Institute, Jiangsu University, China. He received his $\mathrm{PhD}$ degree from Jiangsu Universtiy, China, in 2016. His research interests include vehicle system dynamics. Tel: +86-15952818843; E-mail: sxq@ujs.edu.cn

Yu-Jun Wang, born in 1992, is currently a master candidate at Automotive Engineering Research Institute, Jiangsu University, China.

Ying-Feng Cai, born in 1985, is currently a professor and a $\mathrm{PhD}$ candidate supervisor at Automotive Engineering Research Institute, Jiangsu University, China. Her main research interests include vehicle system dynamics, intelligent vehicles and visual perception.

Pak-Kin Wong, is currently a professor and a $\mathrm{PhD}$ candidate supervisor at Department of Electromechanical Engineering, University of Macau, Taipa, Macau. His main research interests include automotive engines, drive trains and chassis.

Long Chen, born in 1958, is currently a professor and a $\mathrm{PhD}$ candidate supervisor at Automotive Engineering Research Institute, Jiangsu University, China. His main research interests include vehicle system dynamics and advanced vehicle control theory. 
Figures
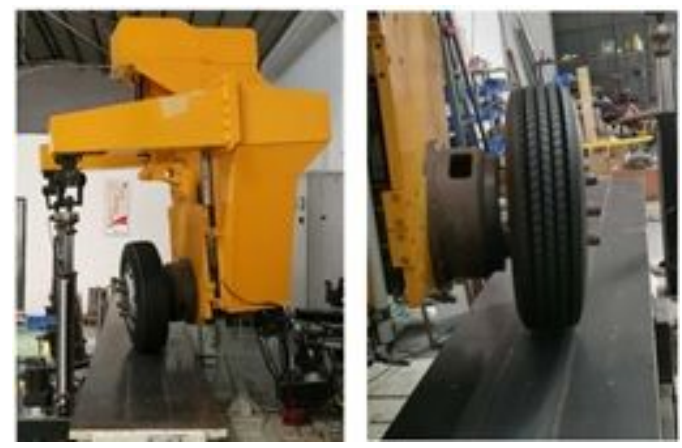

Figure 1

Experimental setup of the tire mechanical characteristics tests.

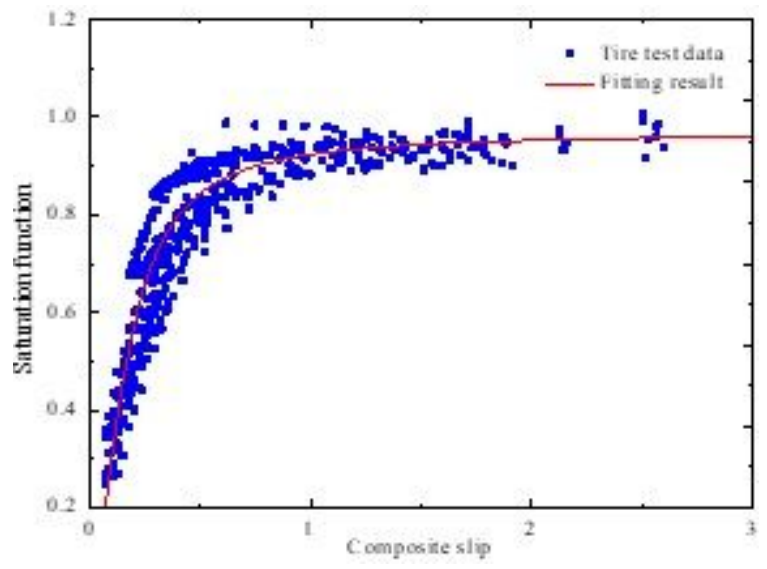

Figure 2

Comparison between the experimental results and the simulation results of the tire force saturation function.

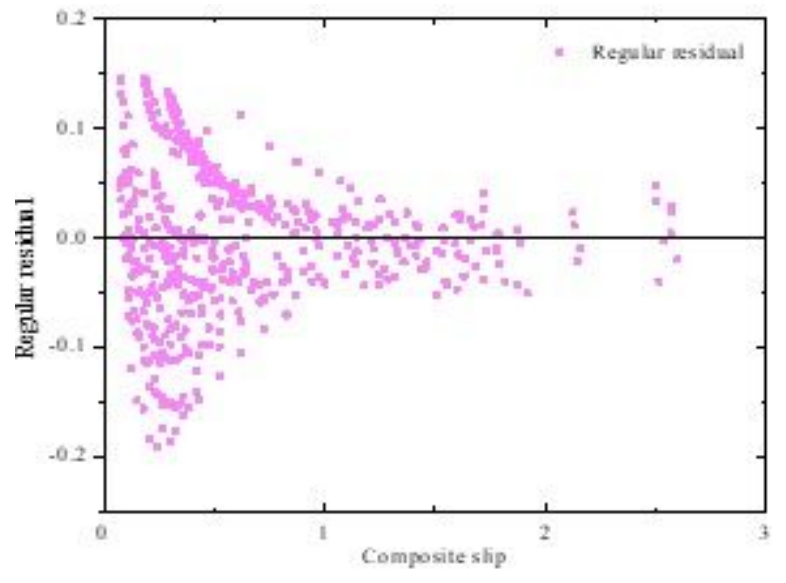

Figure 3

Fitting residual of the tire force saturation function. 


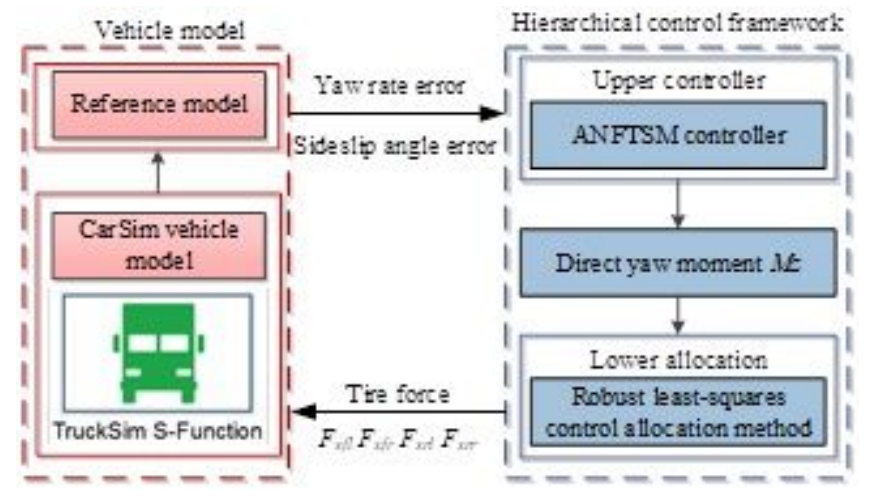

\section{Figure 4}

Hierarchical control framework.

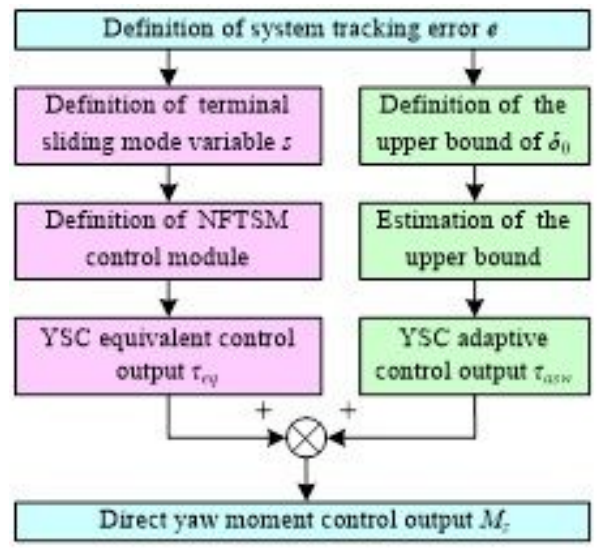

\section{Figure 5}

Control principle of ANFTSM

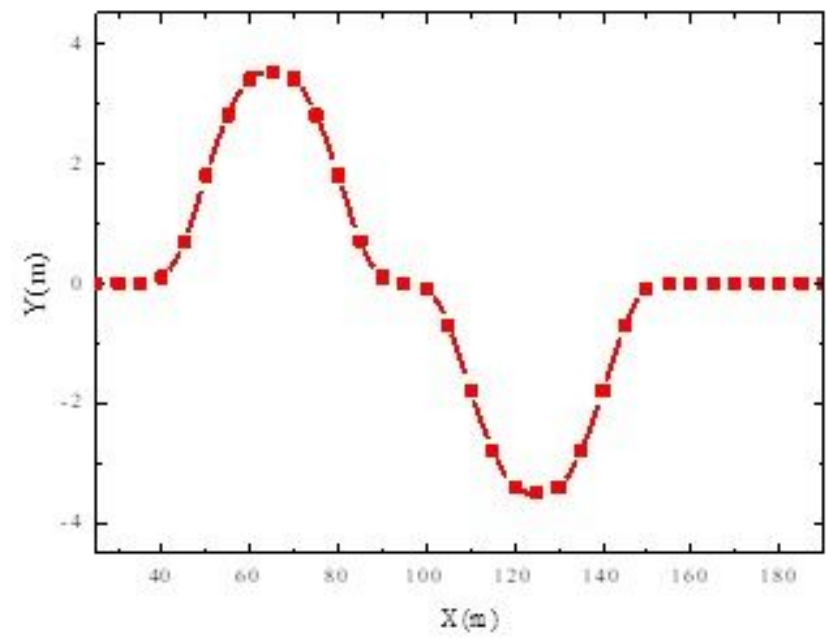

Figure 6

Target trajectory curves. 


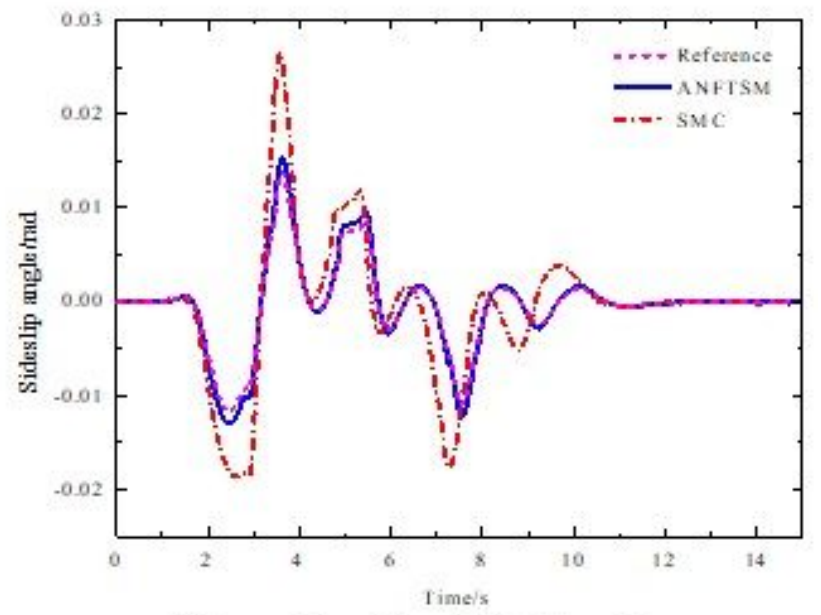

(a) Comparison of the vehicle slip angle

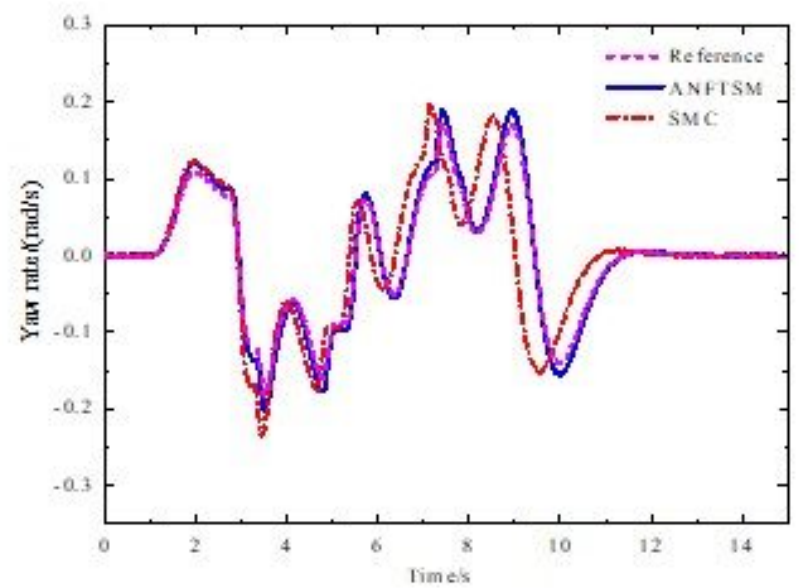

(b) Comparison of the vehicle yaw rate

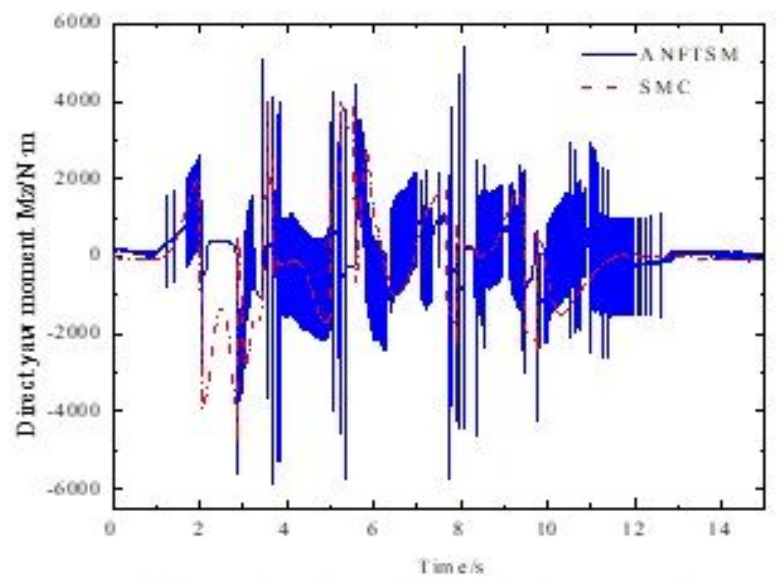

(c) Comparison of the calculated direct yaw moment

Figure 7

Simulation results comparison between the ANFTSM and the SMC $(\mu=0.1)$. 

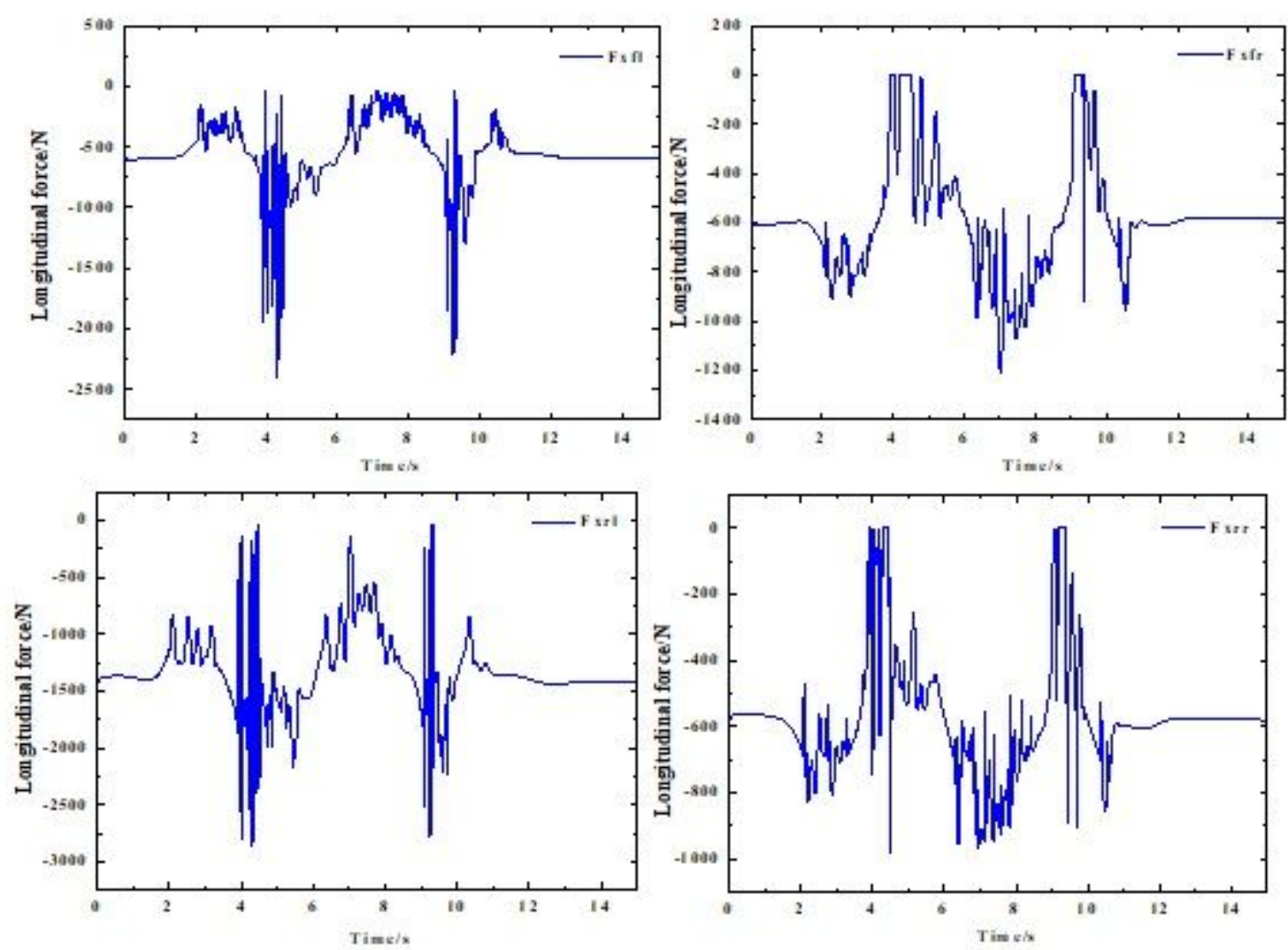

Figure 8

Tire forces allocation results based on the robust least-squares control allocation method $(\mu=0.1)$
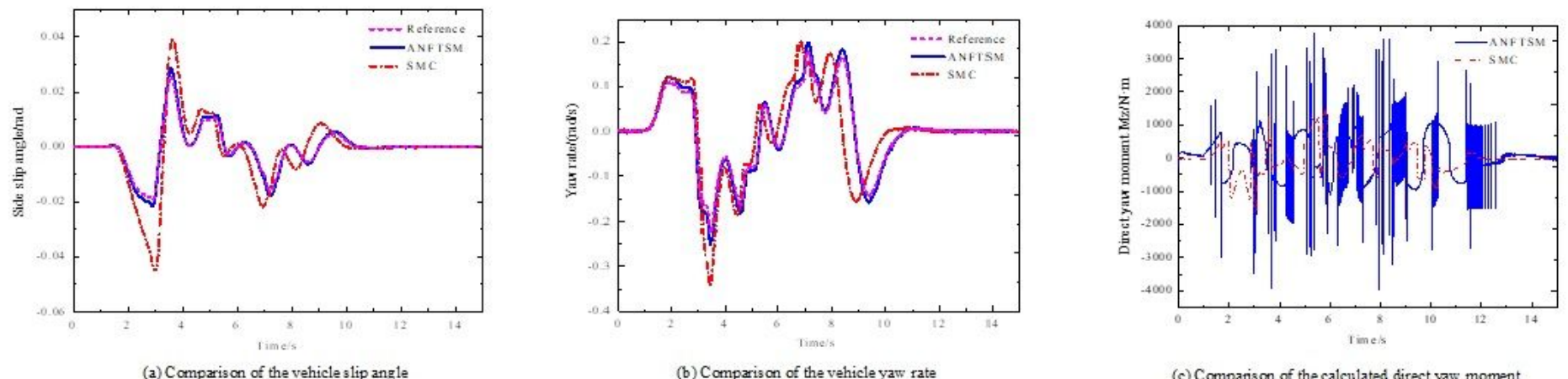

(c) Comparis on of the calculated direct yaw moment

Figure 9

Simulation results comparison between the ANFTSM and the SMC $(\mu=0.3)$. 

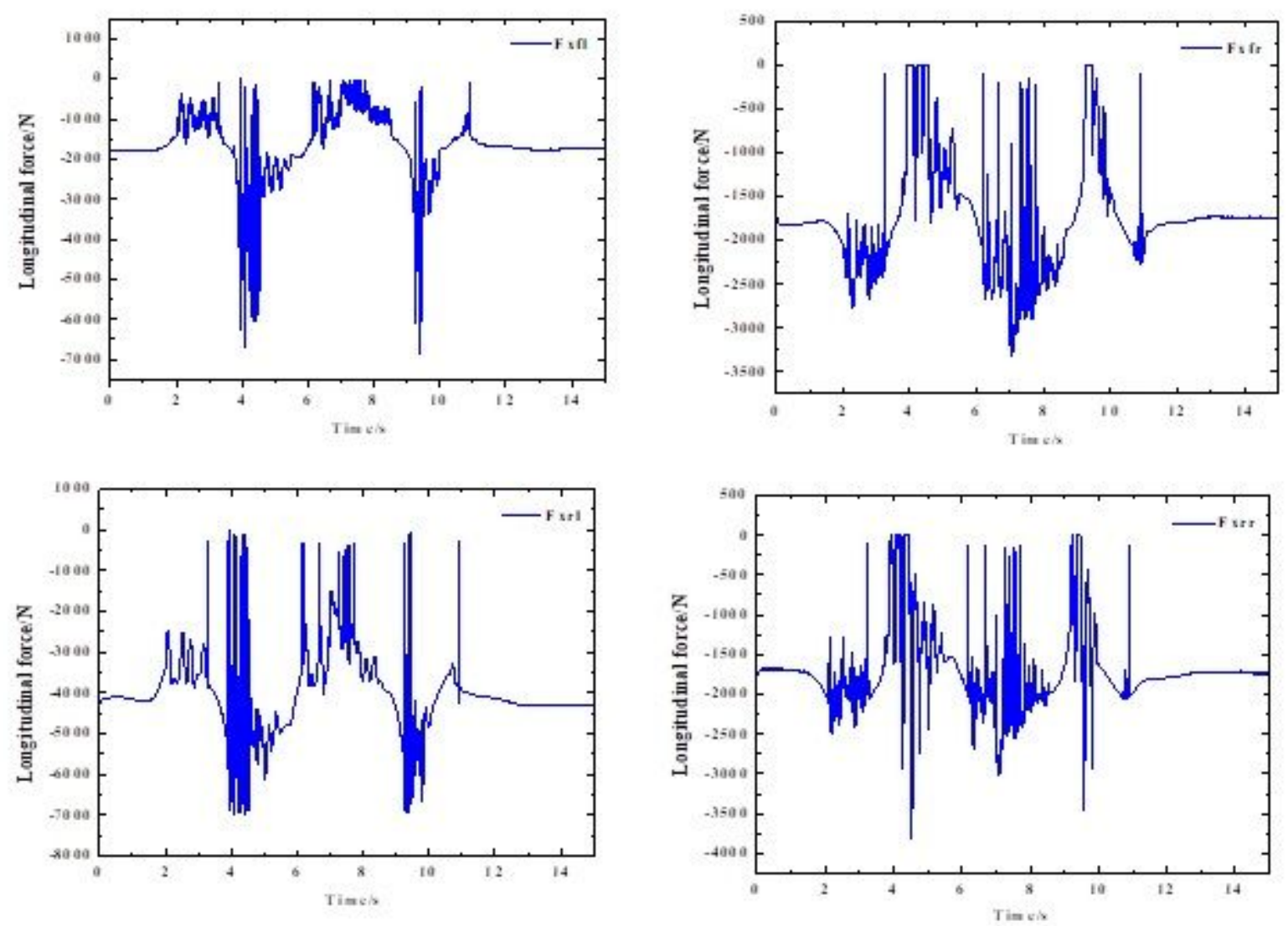

Figure 10

Tire forces allocation results based on the robust least-squares control allocation method $(\mu=0.3)$ 